UVX 2008 (2009) 1-6

(C) EDP Sciences, 2009

DOI: $10.1051 / \mathrm{uvx} / 2009002$

\title{
Une ligne VUV au Serveur Laser du Centre Laser de I'Université Paris-Sud
}

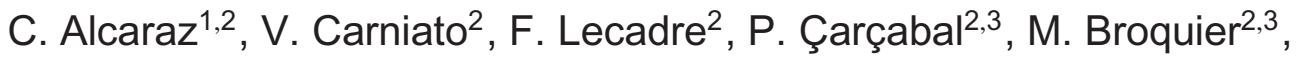 \\ C. Dedonder-Lardeux ${ }^{2,3}$ et C. Jouvet ${ }^{2,3}$ \\ ${ }^{1}$ Laboratoire de Chimie Physique, UMR 8000 CNRS et Université Paris Sud 11, Bât. 350, \\ Centre Universitaire Paris-Sud, 91405 Orsay Cedex, France \\ ${ }^{2}$ Centre Laser de l'Université Paris-Sud, CLUPS, Équipe d'accueil 4127, Université Paris \\ Sud 11, Bât. 106 arrière, Centre Universitaire Paris-Sud, 91405 Orsay Cedex, France \\ ${ }^{3}$ Laboratoire de Photophysique Moléculaire, UPR 3361 CNRS, Bât. 210, Fédération LUMAT, \\ Centre Universitaire Paris-Sud, 91405 Orsay Cedex, France
}

\begin{abstract}
Résumé. Au Centre Laser de l'Université Paris-Sud (CLUPS), un serveur laser (SELA) pour la physicochimie permet d'accueillir des expérimentateurs autour de sources laser nanoseconde synchronisées à 10 $\mathrm{Hz}$ dans des domaines de fréquences variés. Des lasers commerciaux à colorants et OPO pompés par des Nd-YAG couvrent les domaines IR, VIS et UV. Ce domaine a été étendu dans le VUV entre 6 et 19 eV (200$65 \mathrm{~nm})$ par mélange non-linéaire à 4 ondes dans des gaz rares $(\mathrm{Xe}, \mathrm{Kr}, \mathrm{Ar})$ en suivant les deux schémas résonants $\omega_{V U V}=2 \omega_{1} \pm \omega_{2}$ qui s'appuient sur un état excité du gaz rare. Un monochromateur sépare le VUV souhaité des autres fréquences et le refocalise au centre de l'expérience. La génération de VUV accordable a été caractérisée en flux (qq $10^{8}$ à $10^{9}$ photons/pulse) et en pouvoir résolvant $(\mathrm{E} / \Delta \mathrm{E}=500000$ à $15.8 \mathrm{eV})$, ce dernier étant mesuré sur les raies d'autoionisation très fines de l'argon $3 s^{2} 3 p^{5}\left({ }^{2} P_{1 / 2}\right) n s$ couramment appelées ns'. Le pouvoir résolvant de cette source laser VUV et la possibilité de la coupler avec les autres lasers dans des expériences à plusieurs couleurs (IR-VIS-UV + VUV) la rend très intéressante et complémentaire des lignes synchrotron VUV, comme DESIRS sur SOLEIL.
\end{abstract}

\section{INTRODUCTION}

Le domaine du rayonnement ultraviolet du vide (VUV pour Vacuum Ultra-Violet) démarre à des énergies de photon supérieures à $6.2 \mathrm{eV}(\lambda<200 \mathrm{~nm})$. Il est absorbé dans l'air (d'où son nom), en premier lieu à cause des bandes de Schuman-Runge de l'oxygène moléculaire. Le domaine entre 6 et $25 \mathrm{eV}$ correspond à l'énergie nécessaire pour atteindre le 1er potentiel d'ionisation de tout atome ou molécule neutre. Il est donc particulièrement intéressant pour l'étude des états très excités de valence des molécules neutres et pour l'étude des états excités des ions moléculaires jusqu'à leur premier seuil de fragmentation. Le rayonnement VUV est très utilisé pour l'étude des processus d'excitation, de photodissociation, de photoionisation et d'ionisation photodissociative.

Comme il n'existe pas dans ce domaine de sources laser accordables commerciales, on montre ici comment on peut étendre le domaine des lasers commerciaux UV-Vis-IR au domaine VUV de manière continue entre 6.2 et $19.7 \mathrm{eV}(\lambda=200-63 \mathrm{~nm})$. On décrit comment ceci a été implémenté au Centre Laser de l'Université Paris-Sud (CLUPS) pour permettre aux utilisateurs du centre d'utiliser ce rayonnement VUV ou même une combinaison de plusieurs photons de couleurs différentes VUV+ IR-Vis-UV. On donnera les résultats de la caractérisation de la largeur spectrale et du nombre de photons VUV générés, et quelles valeurs de résolution totale on peut espérer en utilisant ces photons avec différentes techniques expérimentales comme par exemple celles utilisées pour l'étude de la photoionisation en phase gazeuse.

Le domaine de rayonnement VUV est aussi couvert par les sources synchrotron, comme SOLEIL à St Aubin, en particulier sur la ligne DESIRS [1]. Le point fort de ce type de sources est sans conteste leur grande accordabilité. On montrera pourquoi les deux types de sources laser et synchrotron sont 
tout à fait complémentaires en termes d'accordabilité, de résolution et de combinaison avec d'autres rayonnements.

\section{GÉNERATION DE RAYONNEMENT LASER VUV PAR MÉLANGE NON-LINÉAIRE DE FRÉQUENCES}

\subsection{Principe}

Pour générer du rayonnement laser accordable d'énergie inférieure à $6.2 \mathrm{eV}(\lambda>200 \mathrm{~nm})$, plusieurs types de lasers commerciaux existent, en particulier des lasers à colorants ou OPO visibles dont la gamme est facilement étendue dans l'UV par mélange non-linéaire d'ordre 2 de fréquences dans des cristaux. Au delà de $6 \mathrm{eV}$, seuls quelques lasers commerciaux existent, mais à des longueurs d'ondes fixes comme les lasers excimères $\operatorname{ArF}(193 \mathrm{~nm})$ ou $F_{2}(157 \mathrm{~nm})$. En ce qui concerne le rayonnement accordable, les cristaux non-linéaires existants, en particulier le BBO, ne permettent pas de descendre bien en dessous de $190 \mathrm{~nm}$ par mélange somme de fréquences (et uniquement si on utilise des fréquences initiales très éloignées). Au delà de $6.2 \mathrm{eV}(\lambda<200 \mathrm{~nm})$, si on veut générer un rayonnement accordable par mélange de fréquences à partir de sources existantes (UV-Vis-IR), il faut utiliser un matériau nonlinéaire transparent au VUV, par exemple un gaz [2]. Comme un gaz est un milieu isotrope, le terme non-linéaire d'ordre $2, \chi^{(2)}$, est nul par symétrie, et le $1^{\text {er }}$ terme non-linéaire non nul qu' on peut utiliser sera celui d'ordre 3. Typiquement, pour générer un photon VUV (jusqu'à $20 \mathrm{eV}$ ), il faut sommer 3 photons UV-Vis comme schématisé sur la Fig. 1 (Panel a). Le processus est plus efficace par plusieurs ordres de grandeur si on utilise les processus résonants schématisés sur la Fig. 1 (panels b et c) dans lesquels les deux premiers photons sont en résonance avec un état excité du gaz. On génère ainsi la fréquence somme $\omega_{V U V}=2 \omega_{1}+\omega_{2}$ (panel b) ou différence $\omega_{V U V}=2 \omega_{1}-\omega_{2}$ (panel c). Par soucis de simplicité, la résonance est atteinte avec deux photons de la même fréquence. On a ainsi à superposer seulement deux faisceaux de couleur différente. Pour un état résonant donné, l'accordabilité du VUV est obtenu en fixant la première fréquence $\omega_{1}$ sur la résonance à 2 photons et en balayant la deuxième fréquence $\omega_{2}$. Ce sont ces deux schémas qui ont été choisis principalement au CLUPS.
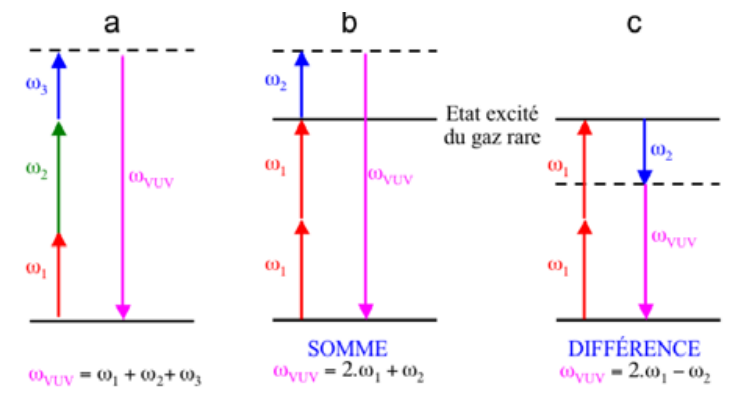

Figure 1. Dans un gaz isotrope, on peut générer un photon VUV par mélange somme ou différence de trois fréquences UV-Vis.

\subsection{Choix du gaz non-linéaire et des fréquences UV-Vis initiales $\omega_{1}$ et $\omega_{2}$}

Pour couvrir toute la gamme VUV, il faut trouver des atomes ou molécules qui possèdent des états résonants à des énergies variées. Les gaz rares (Xe, $\mathrm{Kr}, \mathrm{Ar})$, qui permettent celà, ont été choisis car ils sont très simples à manipuler. L'énergie des états résonants utilisés sont donnés dans la Tab. 1. On a résumé sur la Fig. 2 toute la gamme VUV couverte en fonction du choix du gaz rare, de l'état résonant (caractérisé par la valeur de $\lambda_{1}$ ), du schéma somme ou différence et de l'extension de la deuxième fréquence $\omega_{2}$ (barre verte et bleue). Les deux fréquences $\omega_{1}$ et $\omega_{2}$ initiales sont générées par deux lasers à colorants pulsés $(10 \mathrm{~Hz})$ nanoseconde $(5-10 \mathrm{~ns})$ du fabriquant Quantel (TDL90 pompés par un laser 
Nd-YAG YG981E). L'extension UV standard des lasers TDL90 descend jusqu'à $200 \mathrm{~nm}$, ce qui permet d'atteindre les quatres résonances du Xe et $\mathrm{Kr}$ pour laquelle $\lambda_{1}>200 \mathrm{~nm}$. L'extension UV-Vis 200$750 \mathrm{~nm}$ du $2^{\text {ème }}$ laser à colorant TDL90 qui génère $\omega_{2}$ est symbolisée par la barre verte et bleue sur la Fig. 2 (vert $=$ pompe à $532 \mathrm{~nm}$ et bleue $=$ pompe à $355 \mathrm{~nm})$.

Tableau 1. Etats résonants des gaz rares utilisés dans la génération de VUV par somme et différence de 3 fréquences résonante à 2 photons. Energies tirées de [3].

\begin{tabular}{lllll}
\hline$\lambda_{1}(\mathrm{~nm})$ & $\mathrm{Gaz}$ & Terme spectroscopique & $\mathrm{E}_{\text {Résonance }}\left(\mathrm{cm}^{-1}\right)$ & $\mathrm{E}_{\text {Résonance }}(\mathrm{eV})$ \\
\hline $157.631\left(\lambda_{E x}\right), 220.832\left(\lambda_{1}\right)$ & $\mathrm{Ar}$ & $3 s^{2} 3 p^{5}\left({ }^{2} P_{1 / 2}\right) 4 p^{\prime}[1 / 2]_{0}$ & 108722.6194 & 13.4799 \\
192.749 & $\mathrm{Kr}$ & $4 s^{2} 4 p^{5}\left({ }^{2} P_{3 / 2}\right) 6 p[1 / 2]_{0}$ & 103761.6336 & 12.8648 \\
202.316 & $\mathrm{Kr}$ & $4 s^{2} 4 p^{5}\left({ }^{2} P_{1 / 2}\right) 5 p^{\prime}[1 / 2]_{0}$ & 98855.0698 & 12.2565 \\
212.556 & $\mathrm{Kr}$ & $4 s^{2} 4 p^{5}\left({ }^{2} P_{3 / 2}\right) 5 p[1 / 2]_{0}$ & 94092.8626 & 11.6660 \\
222.568 & $\mathrm{Xe}$ & $5 s^{2} 5 p^{5}\left({ }^{2} P_{1 / 2}\right) 6 p^{\prime}[1 / 2]_{0}$ & 89860.015 & 11.1412 \\
249.629 & $\mathrm{Xe}$ & $5 s^{2} 5 p^{5}\left({ }^{2} P_{3 / 2}\right) 6 p[1 / 2]_{0}$ & 80118.962 & 9.9335 \\
\hline
\end{tabular}

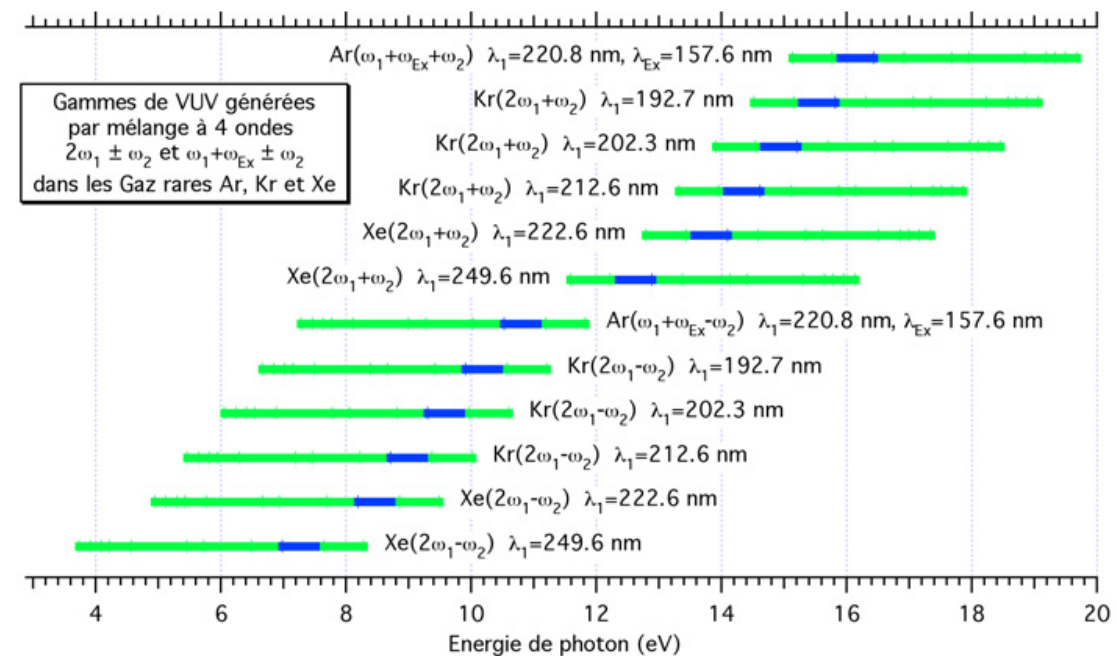

Figure 2. Gamme d'énergie de photon VUV généré par mélange somme ou différence dans les gaz rares Xe, $\mathrm{Kr}$ ou Ar.

Le 1er laser à colorant TDL90 a été modifié pour générer également $\lambda_{1}=192.7 \mathrm{~nm}$ qui permet d'atteindre la $5^{\text {ème }}$ résonance (plus haute résonance du Kr de la Fig. 2) par mélange du $4^{\text {ème }}$ harmonique du YAG à $266 \mathrm{~nm}$ et de visible proche de $700 \mathrm{~nm}$.

La plupart de ces schémas ont déjà été mis en œuvre. On voit néanmoins qu'au voisinage de $11.5 \mathrm{eV}$, il existe un petit domaine non couvert par ces cinq résonances. Pour couvrir cette zone et pour ne pas avoir à changer radicalement de configuration dans cette gamme, on est en cours d'implémentation d'une dernière configuration correspondant à une résonance de Ar. Contrairement aux autres résonances, on ne peut l'atteindre avec deux photons identiques $\lambda_{1}$ qui seraient de trop courte longueur d'onde dans l'UV $(184 \mathrm{~nm})$ pour pouvoir être générés par somme de fréquence. On utilise plutôt le schéma proposé par Rupper et Merkt [4] dans lequel un photon $\omega_{E x}$ de longueur d'onde $157 \mathrm{~nm}$ est généré par un laser à excimère $\mathrm{F}_{2}$, et le photon $\omega_{1}$ de longueur d'onde proche de $220.8 \mathrm{~nm}$ nécessaire pour atteindre alors la résonance est généré par le $1^{\text {er }}$ laser à colorant. Le $2^{\text {ème }}$ laser à colorant joue le même rôle que dans les autres schémas et est balayé pour générer du VUV accordable somme ou différence $\omega_{V U V}=\omega_{1}+\omega_{E x} \pm \omega_{2}$. C'est un schéma plus difficile à mettre en œuvre car il faut superposer trois 
faisceaux de couleur différente, et l'un d'entre eux, le $157 \mathrm{~nm}$, ne se propage pas dans l'air mais seulement dans le vide ou bien dans un gaz non-absorbant $\left(\mathrm{N}_{2}, \mathrm{Ar}\right)$.

\subsection{Mise en œuvre}

La Fig. 3 montre les trois pièces composant le Serveur Laser (SELA) du CLUPS, l'agencement des lasers et des dispositifs expérimentaux dans ces salles. Les lasers commerciaux sont installés dans la salle laser et les faisceaux lasers sont transportés dans les salles d'expériences à travers des fenêtres, ce qui permet une grande latitude d'utilisation des différents rayonnements dans chaque expérience.

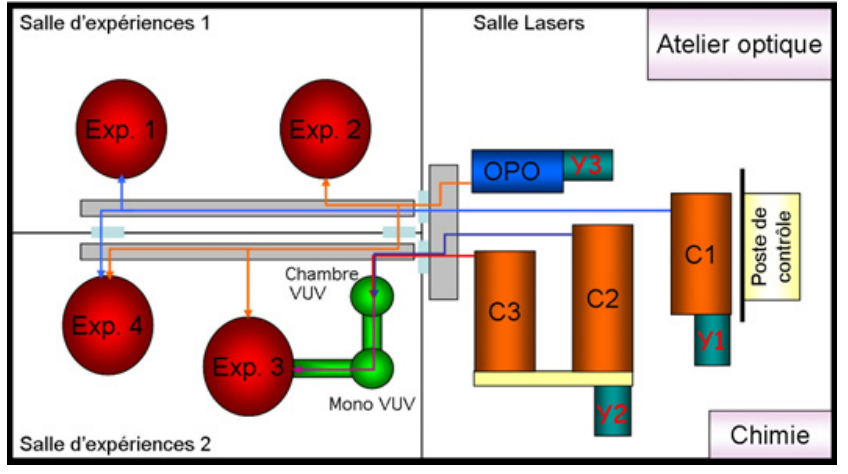

Figure 3. Le Serveur Laser (SELA) du CLUPS.

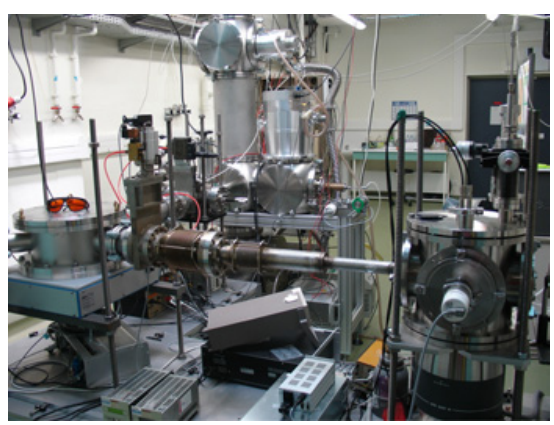

Figure 4. Chambre et monochromateur VUV et dispositif expérimental.

Pour générer le rayonnement VUV, on utilise les lasers à colorants $\mathrm{C} 2\left(\omega_{1}\right)$ et $\mathrm{C} 3\left(\omega_{2}\right)$ pompés par le laser Nd-YAG Y2. Les faisceaux $\omega_{1}$ et $\omega_{2}$ sont transportés vers le dispositif VUV dans la salle d'expérience 2, où ils sont alignés, superposés puis focalisés dans la chambre VUV (en vert sur la Fig. 3). On a mis au point deux modes de production de VUV, en cellule fermée et en jet libre. Pour des énergies inférieures à $11-12 \mathrm{eV}$, on utilise la configuration en cellule qui est plus efficace. Le gaz rare est alors contenu par des fenêtres en $\mathrm{MgF}_{2}$ ou en $\mathrm{LiF}$ qui transmettent le VUV jusqu'à pratiquement 11 et $12 \mathrm{eV}$ respectivement. Pour des énergies plus grandes, il n'existe pas de matériaux suffisemment transparent au VUV et on doit utiliser alors la configuration en jet libre qui est généré par une vanne pulsée (General Valve) à $10 \mathrm{~Hz}$ et synchronisée avec $\omega_{1}$ et $\omega_{2}$. On voit sur la droite de la Fig. 4 , la chambre VUV et le dispositif de réglage $\mathrm{XYZ}$ du jet moléculaire qui est vertical. Les faisceaux sont focalisés à très courte distance de l'orifice de la tuyère du jet et cette distance est optimisée sur l'intensité du VUV généré.

Les fréquences initiales $\left(\omega_{1}\right.$ et $\left.\omega_{2}\right)$ et $\operatorname{VUV}\left(3 \omega_{1}, 2 \omega_{1}+\omega_{2}, 2 \omega_{1}-\omega_{2}\right)$ sont séparées par un monochromateur VUV (Jobin-Yvon) composé d'un réseau torique de $600 \mathrm{tr} / \mathrm{mm}$ et visible sur la gauche de la photo (Fig. 4). Le rayonnement VUV choisi est refocalisé par ce même réseau au centre du dispositif expérimental visible en arrière-plan sur la Fig. 4.

\subsection{Caractérisation du rayonnement VUV généré : flux et finesse spectrale}

Pour mesurer le flux de VUV généré au niveau du disposif expérimental où on va l'utiliser, on a installé une plaque d'or qui, par effet photoélectrique, emet des électrons avec une efficacité dépendant de l'énergie de photon VUV et proportionnelle au nombre de photons. Le rendement photoélectrique en fonction de l'énergie de photon étant connu, on déduit du courant mesuré le nombre absolu de photons générés. On a trouvé des valeurs typiquement entre $10^{9}$ et $10^{10}$ photons / sec, c'est à dire entre $10^{8}$ et $10^{9}$ photons / impulsion. 


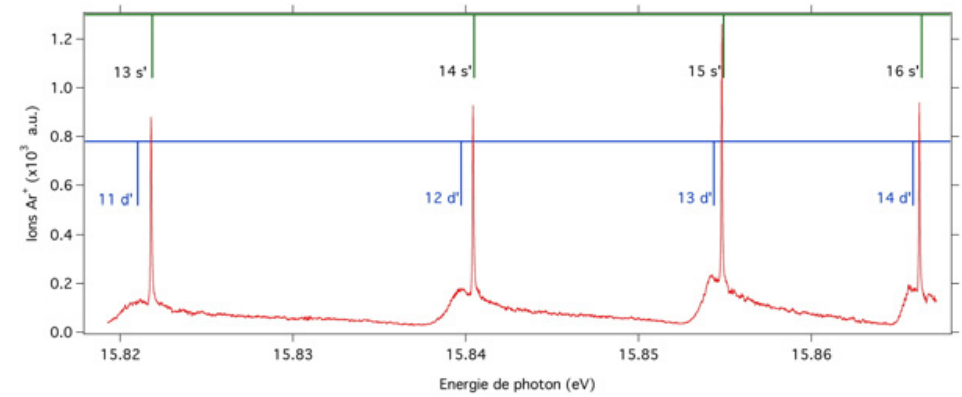

Figure 5. Spectre de photoionisation de Ar à 1 photon avec le laser VUV du CLUPS : résonances d'autoionisation nd' $\left(3 s^{2} 3 p^{5}\left({ }^{2} P_{1 / 2}\right) n d\right)$ et ns' $\left(3 s^{2} 3 p^{5}\left({ }^{2} P_{1 / 2}\right) n s\right)$ de Ar entre les deux $1^{\text {ers }}$ états ${ }^{2} P_{3 / 2}$ et ${ }^{2} P_{1 / 2} d e A r^{+}$.

Pour caractériser, la finesse spectrale du rayonnement VUV, on a utilisé des raies d'autoionisation de l'argon dont la largeur naturelle est très fine et documentée dans la littérature. L'expérience consiste à détecter les ions $\mathrm{Ar}^{+}$issus de la photoionisation de $\mathrm{Ar}$ autour de 15.8 eV. Dans cette zone entre les deux premiers états de $\mathrm{Ar}^{+},{ }^{2} P_{3 / 2}$ et ${ }^{2} P_{1 / 2}$, la forme complexe du rendement d'ions que l'on peut voir sur la Fig. 5 résulte de l'interférence entre cinq canaux correspondant à des séries de Rydberg de Ar convergeant vers les états ${ }^{2} P_{3 / 2}$ ou ${ }^{2} P_{1 / 2}$ de $\mathrm{Ar}^{+}$. Le VUV a été généré dans du Xe en utilisant la résonance à $89860 \mathrm{~cm}^{-1}$ avec $\lambda_{1}=222.6 \mathrm{~nm}$ et $\lambda_{2}=261-265 \mathrm{~nm}$. Les pics très grands et fins sont ceux qui nous intéressent ici et apparaissent au voisinage de chaque état de Rydberg de la série $3 s^{2} 3 p^{5}\left({ }^{2} P_{1 / 2}\right) n s^{\prime}[1 / 2]_{1}$. Leur largeur naturelle est très petite et diminue avec $\mathrm{n}\left(\mathrm{en} 1 / \mathrm{n}^{3}\right)$ comme on peut le voir sur les enregistrements plus précis de la Fig. 6. La mesure de la largeur de chacun des pics permet après déconvolution de la largeur naturelle des raies de déduire la finesse spectrale du rayonnement VUV. On trouve une largeur d'environ $0.26 \mathrm{~cm}^{-1}$ dans cette gamme d'énergie autour de $15.8 \mathrm{eV}\left(127000 \mathrm{~cm}^{-1}\right)$, ce qui fait un $\mathrm{E} / \Delta \mathrm{E}$ proche de 500000.
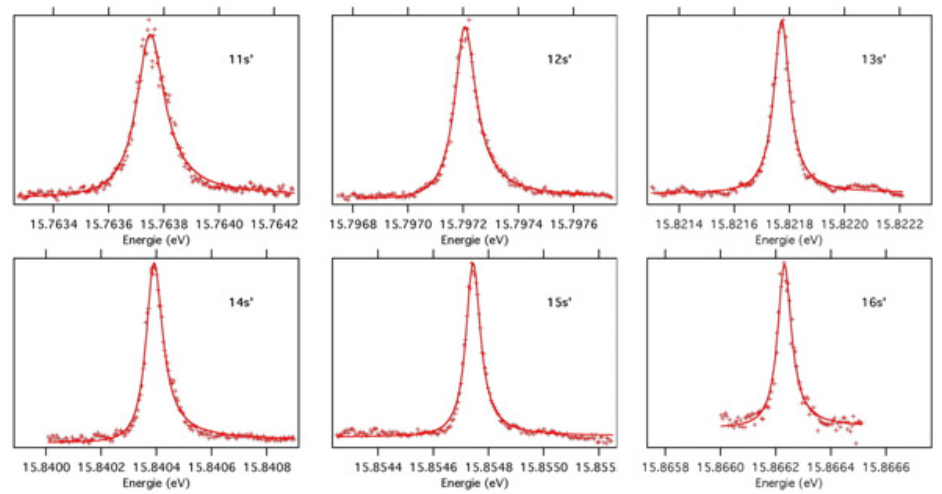

Figure 6. Résonances d'autoionisation ns' $\left(3 s^{2} 3 p^{5}\left({ }^{2} P_{1 / 2}\right) n s\right)$ de Ar pour $\mathrm{n}=11-16$.

\section{APPLICATIONS : COMPLÉMENTARITÉ DES RAYONNEMENTS LASER ET SYNCHROTRON}

Des premiers utilisateurs sont venus faire des expériences dans différents domaines :

- Photoionisation du bis-benzoxazole : M. Borowicz et J. Sepiol (Polish Academy of Science Varsovie), A. Zehnacker (LPPM)

- Photoionisation à deux couleurs IR + VUV (sélection d'un conformère) : H. Kang (U. Sejou, Corée), C. Jouvet, C. Dedonder, M. Broquier (LPPM) 
- Mesure ZEKE du potentiel d'ionisation de la fenchone : C. Dedonder, A. Zehnacker, M. Broquier, C. Jouvet (LPPM)

- Photoionisation de radicaux $\left(\mathrm{C}_{3} \mathrm{H}_{5}, \mathrm{CH}_{3}\right)$ produits par pyrolyse éclair : C. Alcaraz (LCP)

- Photoionisation de carbènes (bi-radicaux) produits par pyrolyse : B. Noller (U. Würzburg)

- Section efficace absolue de photoionisation de radicaux $\left(\mathrm{CH}_{3}\right)$ produits par pyrolyse ou photolyse @ 266 nm de $\mathrm{CH}_{3} \mathrm{I}$ : B. Gans, S. Boyé-Peronne, S. Douin, D. Gauyacq (LPPM)

La particularité du Serveur Laser (SELA) qui le rend très attrayant est la réunion dans un même lieu de rayonnements laser de domaines très variés (VUV, UV, Vis, IR) qui peuvent être utilisés de manière synchronisée $(10 \mathrm{~Hz}, 10 \mathrm{~ns})$ dans une même expérience à plusieurs couleurs.

Dans le domaine VUV, l'autre avantage du SELA est la grande finesse spectrale du rayonnement laser généré par mélange de fréquence $\left(\Delta \mathrm{E}=0.26 \mathrm{~cm}^{-1}\right.$ et $\mathrm{E} / \Delta \mathrm{E}=500000$ à $\left.15.8 \mathrm{eV}\right)$. De plus, avec des sources VUV laser, on peut utiliser des techniques d'ionisation par champ (PFI/ZEKE). Dans ce cas, et si on contrôle proprement les champs électriques nécessaires à la technique, la résolution totale expérimentale peut être très proche de la finesse spectrale seule $\left(\Delta \mathrm{E}=0.26 \mathrm{~cm}^{-1}\right)$ et en tout cas inférieure au $\mathrm{cm}^{-1}$, ce qui permet de distinguer les niveaux d'énergie rotationels.

Le rayonnement synchrotron (RS) est un outil puissant qui délivre également du rayonnement dans le domaine VUV. Ses points les plus forts sont son extrème accordabilité, son intensité et sa grande stabilité. Même si cela est possible, il est par contre plus complexe de synchroniser des sources lasers externes à coupler avec le RS dans des expériences à plusieurs couleurs. Les lignes modernes, comme par exemple la ligne DESIRS au synchrotron SOLEIL [1] permettent maintenant de produire un rayonnement VUV monochrome avec un pouvoir résolvant E/AE voisin de 200000 [5]. Associé à des spectromètres de photoélectrons modernes [6], on arrive à une résolution totale proche du $\mathrm{meV}\left(8 \mathrm{~cm}^{-1}\right)$ permettant de distinguer les niveaux d'énergie vibrationels. En revanche, avec d'autres techniques expérimentales comme par exemple l'absorption, c'est la finesse spectrale qui est le terme dominant dans la résolution totale. Avec un monochromateur, on peut ainsi s'approcher d'un pouvoir résolvant $\mathrm{E} / \Delta \mathrm{E}$ proche de 200000 . Un pouvoir résolvant supérieur $(\mathrm{E} / \Delta \mathrm{E}=700000)$ a même été atteint avec le spectromètre VUV à transformée de Fourier installé sur DESIRS (voir la contribution de D. Joyeux dans ce volume). On voit donc que le SELA et SOLEIL sont deux outils très complémentaires proposés aux utilisateurs.

\section{Références}

[1] DESIRS: http://www.synchrotron-soleil.fr/portal/page/portal/Recherche/LignesLumiere/DESIRS

[2] J.W. Hepburn, Vacuum Ultraviolet Photoionization and Photodissociation of Molecules and Clusters (World Scientific Publishing Co. Pte. Ltd., Singapore, 1991) pp. 435

[3] NIST2008: Ralchenko, Yu., Kramida, A.E., Reader, J., and NIST ASD Team (2008). NIST Atomic Spectra Database (version 3.1.5), http://physics.nist.gov/asd3

[4] P. Rupper and F. Merkt, Rev. Sci. Instrum. 75, (2004) 613.

[5] L. Nahon, C. Alcaraz, J.-L. Marlats, B. Lagarde, F. Polack, R. Thissen, D. Lepère, and K. Ito, Rev. Sci. Intrum. 72, (2001) 1320.

[6] G.A. Garcia, L. Nahon, C.J. Harding, E.A. Mikajlo, and I. Powis, Rev. Sci. Intrum. 76, (2005) 053302 . 